\title{
Immune Thrombocytopenia in Chronic Lymphocytic Leukemia
}

\section{Amelia Maria Gaman ${ }^{1,2 *}$ and Mihnea-Alexandru Gaman ${ }^{3}$}

${ }^{1}$ Department of Pathophysiology, University of Medicine and Pharmacy of Craiova, Romania

${ }^{2}$ Department of Pathophysiology, Filantropia City Hospital Craiova, Romania

${ }^{3}$ University of Medicine and Pharmacy, Bucharest, Romania

\begin{abstract}
Introduction: Chronic lymphocytic leukemia (CLL) is a malignant proliferation of mature, differentiated B-lymphocytes associated in 1-5 \% of cases with immune thrombocytopenia (ITP). The effect of ITP on the clinical outcome and survival of patients with CLL is controversial.
\end{abstract}

Materials and methods: The aim of the study was to evaluate the level of oxidative stress in patients with CLL and ITP and how this complication influences the patient's survival.

We studied 84 patients with CLL hospitalized in the Clinic of Hematology from Craiova (Romania) between 2007 and 2012 .The global oxidative status at diagnosis of CLL and in the presence of ITP by FORT (Free Oxygen Radicals Testing) and FORD (Free Oxygen Radical Defence) tests and the median overall survival in patients with CLL - stage C immune disease compared to stage $C$-infiltrative disease were evaluated.

Results and discussion: All patients with CLL had low FORD values and high FORT values and there were no significant differences of oxidative stress between patients with CLL and ITP compared to patients with CLL without ITP. Oxidative stress did not play a role in the appearance of ITP in CLL. The patients with CLL-stage C immune disease had a longer survival than the patients with CLL-stage $C$ infiltrative disease.

Keywords: CLL; Immune thrombocytopenia; Oxidative stress; Survival; FORD; FORT

\section{Introduction}

Chronic lymphocytic leukemia (CLL) is a malignant proliferation of mature, differentiated lymphocytes, mostly of B-cell origin with distinctive immunophenotype: ie, SmIg, CD5+, CD19+, CD20+, $\mathrm{CD} 23+$ in peripheral blood, bone marrow, lymph nodes, other lymphoid tissues [1]. CLL is sometimes associated with autoimmune cytopenia: autoimmune hemolytic anemia (5-25\% of cases) and immune thrombocytopenia (1-5\% of cases) [2-4]. In CLL, immune thrombocytopenia is associated with unmutated $\mathrm{IgVH}$ (a positive direct antiglobulinic test) and the development of autoimmune hemolytic anemia [5]. The effect of immune thrombocytopenia on the clinical outcome and survival of patients with CLL is controversial $[2,4,5]$.

All biochemical reactions occuring in human cells involve the transfer of electrons and therefore Reactive Oxygen Species (ROS) are formed. They also have beneficial effects (mitochondrial function, immune response) but when they become excessive, they are significantly destructive and attack fundamental cellular components (DNA, lipids, proteins). Oxidative stress, defined as an imbalance between ROS and antioxidants, is involved in many physiological and pathophysiological processes: intercellular communication, proteinkinase activity, mitochondrial structure and function, genic expression, cell growth, immune response, apoptosis, carcinogenesis etc. [6-8]. In the secondary immune thrombocytopenia from HIV and HCV infections, specific antibodies for GP49-66 are involved, antibodies which have the ability to induce reactive oxygen species through the activation of 12-lipooxygenase and NADPH-oxidase, leading to complement-independent platelet fragmentation $[5,9]$.

\section{Materials and Methods}

The aim of the study was to evaluate the level of oxidative stress in patients with CLL and immune thrombocytopenia and how this complication influences the patient's survival. We studied 84 patients with CLL hospitalized in the Clinic of Hematology from Craiova (Romania) between 2007 and 2012 (informed consent obtained). The patients were distributed by age, sex, environmental medium, Binet staging. The diagnosis of CLL was made according to the national protocol and whenever possible the diagnosis was confirmed by flowcytometry. The diagnosis of immune thrombocytopenia was based on the presence on unexplained fall in platelet count to $<100 \times 10^{9} / \mathrm{L}$ and on more than two indirect parameters: evidence of normal bone marrow function (normal or increased megakaryocytes in bone marrow), no splenomegaly and no chemotherapy within the last month. Patients had stage C Binet infiltrative when platelet counts to $<100 \times 10^{9} / \mathrm{L}$ and bone marrow aspirate revealed more than $80 \%$ lymphocytes. Other biological parameters determined were: haemoglobin value, reticulocyte count, leukocyte count and leukocyte formula, peripheral blood smear, bone marrow smear, erythrocytes sedimentation rate, unmutated $\mathrm{IgVH}$ in some cases, bilirubin levels, lactate dehydrogenase and serum $\beta 2$ microglobuline. The antileukemic treatment was represented by chlorambucil+Prednisone, CVP-regimen (Cyclophosphamide+Oncov in+Prednisone), Fludarabine alone, RFC-regimen (Rituximab+Fluda rabine+Cyclophosphamide) or alemtuzumab (anti CD52 monoclonal antibody). The global oxidative status was evaluated at all patients at the diagnosis of CLL and in the presence of immune thrombocytopenia using a CR3000 analyser (Callegari S.p.A). Both free oxygen radicals

*Corresponding author: Amelia Maria Gaman, Department of Pathophysiology, University of Medicine and Pharmacy of Craiova, Romania, Tel: 40770684146; E-mail: gamanamelia@yahoo.com

Received December 02, 2013; Accepted February 18, 2014; Published February 24, 2014

Citation: Gaman AM,Gaman MA (2014) Immune Thrombocytopenia in Chronic Lymphocytic Leukemia. J Blood Disorders Transf 5: 198. doi: 10.4172/2155-9864.1000198

Copyright: (C) 2014 Gaman AM, et al. This is an open-access article distributed under the terms of the Creative Commons Attribution License, which permits unrestricted use, distribution, and reproduction in any medium, provided the original author and source are credited. 
Citation: Gaman AM,Gaman MA (2014) Immune Thrombocytopenia in Chronic Lymphocytic Leukemia. J Blood Disorders Transf 5: 198. doi: 10.4172/2155-9864.1000198

Page 2 of 4

and antioxidant status were evaluated by FORT (Free Oxygen Radicals testing) and FORD (Free Oxygen Radical Defence) tests from a single drop of capillary blood. The FORT test is a colorimetric test based on the ability of transition metals (iron) to catalyse the breakdown of hydroperoxides into derivative radicals according to the Fenton reaction. Once formed in cells, $\mathrm{ROOH}$ maintain their chemical reactivity and oxidative capacity to generate proportional amounts of alkoxyl and peroxyl radicals which are preferentially trapped by a suitably buffered chromogen and develop, at $37^{\circ} \mathrm{C}$, a coloured fairly long-lived radical cation photometrically detectable. The intensity of the colour correlates directly with the quantity of radical compounds and can be related to the oxidative status of the sample. The normal ranges of FORT are up to 310 Fort units or $2.3 \mathrm{mmol} / \mathrm{L}_{2} \mathrm{O}_{2}$. The FORD test is a colorimetric test based on the ability of antioxidants present in plasma to reduce a preformed radical cation. A stable and colored cation detectable photometrically at $505 \mathrm{~nm}$ is formed in the presence of an acidic buffer, at $\mathrm{pH}$ of 5.2, and a suitable oxidant, $\mathrm{FeCl}_{3}$. Antioxidant compounds present in the sample reduce the radical cation of the chromogen quenching the color and producing a decoloration of the solution proportional to their amount in the sample. The normal ranges of FORD are 1.07-1.53 mmol/L. Statistical analysis was performed and a $\mathrm{P}$ value $\leq 0.05$ was considered significant.

\section{Results and Discussion}

The median age of the patients with CLL was of 65 years; the sex ratio $\mathrm{M} / \mathrm{F}=1.62$, revealed a male predominance. Repartition on the stage of disease showed: CLL-stage $\mathrm{A}=23$ patients, CLL-stage $\mathrm{B}=$ 30 patients, CLL-stage $C=31$ patients (Table 1 ). In $31 \%$ of cases, the diagnosis was confirmed by flowcytometry. The patients were treated with corticosteroids alone $=4$ cases, chlorambucil + Prednisone $=16$

\begin{tabular}{|c|c|c|c|c|c|c|c|c|c|}
\hline No. pts & Age (years) & Gender & CLL stage & $\mathrm{Hb} \times 10^{9} / \mathrm{L}$ & $W B C \times 10^{9} / L$ & PIt $\times 10^{9} / L$ & $\begin{array}{l}\text { FORDvalues } \\
\text { (mmol/L) }\end{array}$ & $\begin{array}{l}\text { FORTvalues } \\
\text { (mmol/L) }\end{array}$ & $\begin{array}{c}\text { Survival } \\
\text { (years) }\end{array}$ \\
\hline 1. & 58 & $\mathrm{M}$ & A & 11.9 & 23.1 & 252 & 0.98 & 2.8 & 7.8 \\
\hline 2. & 72 & $M$ & C & 10.3 & 40.9 & 102 & 0.62 & 3.1 & 3.1 \\
\hline 3 & 63 & M & B & 12.1 & 54.9 & 368 & 0.73 & 3.0 & 6.1 \\
\hline 4. & 67 & $M$ & C & 10.9 & 31.8 & 74 & 0.39 & 2.9 & 6.6 \\
\hline 5. & 51 & $M$ & B & 12.8 & 65.6 & 343 & 0.84 & 2.6 & 8.2 \\
\hline 6. & 79 & $\mathrm{~F}$ & $B$ & 11.4 & 29.7 & 322 & 0.59 & 3.3 & 5.3 \\
\hline 7. & 66 & $\mathrm{~F}$ & A & 12.2 & 33.8 & 245 & 0.57 & 2.7 & 9.2 \\
\hline 8. & 64 & $\mathrm{~F}$ & A & 12.4 & 36.2 & 336 & 0.86 & 2.4 & 14.6 \\
\hline 9. & 81 & $M$ & B & 12.0 & 41.8 & 265 & 0.94 & 3.6 & 3.2 \\
\hline 10. & 49 & $\mathrm{M}$ & B & 12.3 & 48.4 & 373 & 0.82 & 3.4 & 7.9 \\
\hline 11. & 62 & $\mathrm{~F}$ & A & 11.6 & 25.3 & 381 & 1.02 & 2.5 & 12.2 \\
\hline 12. & 68 & $\mathrm{~F}$ & C & 10.9 & 38.2 & 104 & 0.67 & 3.2 & 5.5 \\
\hline 13. & 59 & $\mathrm{~F}$ & B & 11.4 & 45.8 & 264 & 0.56 & 2.7 & 6.8 \\
\hline 14 & 71 & $M$ & B & 11.6 & 57.5 & 364 & 0.83 & 3.6 & 4.5 \\
\hline 15. & 68 & $M$ & C & 11.0 & 36.6 & 98 & 0.56 & 2.7 & 4.8 \\
\hline 16 & 62 & $\mathrm{M}$ & A & 12.3 & 27.9 & 372 & 0.85 & 2.5 & 12.1 \\
\hline 17. & 73 & $\mathrm{~F}$ & C & 9.7 & 49.3 & 80 & 0.41 & 3.3 & 2.9 \\
\hline 18. & 57 & $M$ & B & 12.6 & 48.9 & 298 & 0.84 & 3.6 & 6.8 \\
\hline 19. & 69 & $\mathrm{~F}$ & C & 10.8 & 53.1 & 92 & 0.71 & 3.8 & 5.9 \\
\hline 20. & 61 & $M$ & B & 12.5 & 57.3 & 274 & 0.62 & 3.4 & 4.6 \\
\hline 21. & 74 & $M$ & C & 9.6 & 44.2 & 104 & 0.54 & 2.6 & 2.8 \\
\hline 22. & 56 & $M$ & B & 12,4 & 46.9 & 365 & 0.86 & 3.8 & 5.6 \\
\hline 23. & 72 & $\mathrm{~F}$ & C & 9.4 & 43.1 & 88 & 0.66 & 3.1 & 2.5 \\
\hline 24. & 58 & $M$ & B & 12.7 & 44.6 & 382 & 0.92 & 3.4 & 6.9 \\
\hline 25. & 66 & $M$ & C & 8.0 & 30.2 & 68 & 0.84 & 3.5 & 6.9 \\
\hline 26. & 64 & $M$ & $A$ & 12.0 & 36.2 & 363 & 0.56 & 2.8 & 10.3 \\
\hline 27. & 70 & $M$ & C & 10.4 & 46.0 & 94 & 0.58 & 2.9 & 3.4 \\
\hline 28 & 60 & $\mathrm{~F}$ & A & 12.2 & 29.8 & 366 & 0.98 & 2.4 & 14.2 \\
\hline 29. & 47 & $M$ & B & 12.4 & 56.9 & 343 & 0.64 & 2.9 & 6.5 \\
\hline 30. & 83 & $M$ & A & 12.1 & 38.3 & 274 & 0.46 & 3.7 & 3.4 \\
\hline 31. & 70 & $M$ & $C$ & 10.8 & 65.3 & 80 & 0.49 & 3.1 & 4.5 \\
\hline 32. & 59 & $\mathrm{~F}$ & B & 11.9 & 37.9 & 246 & 0.69 & 3.4 & 6.8 \\
\hline 33. & 63 & $\mathrm{~F}$ & A & 12.3 & 27.0 & 386 & 0.87 & 3.1 & 8.2 \\
\hline 34. & 68 & $\mathrm{~F}$ & B & 11.4 & 43.7 & 342 & 0.83 & 3.7 & 4.9 \\
\hline 35. & 74 & $M$ & $C$ & 9.2 & 47.1 & 114 & 0.55 & 2.5 & 2,7 \\
\hline 36. & 56 & $\mathrm{~F}$ & A & 11.8 & 29.2 & 394 & 0.56 & 2.4 & 19.1 \\
\hline 37. & 56 & $M$ & A & 12.4 & 34.6 & 383 & 0.77 & 2.7 & 11.1 \\
\hline 38 & 74 & $M$ & $C$ & 10.6 & 67.2 & 78 & 0.72 & 3.4 & 2.8 \\
\hline 39. & 51 & $\mathrm{~F}$ & B & 11.8 & 41.3 & 374 & 0.84 & 3.8 & 4.6 \\
\hline 40. & 79 & $M$ & B & 11.9 & 58.5 & 376 & 0.52 & 3.8 & 3.6 \\
\hline 41. & 68 & $M$ & A & 12.0 & 33.4 & 342 & 0.76 & 3.2 & 9.3 \\
\hline 42. & 62 & $\mathrm{~F}$ & B & 11.6 & 48.5 & 283 & 0.99 & 3.6 & 5.8 \\
\hline
\end{tabular}


Citation: Gaman AM,Gaman MA (2014) Immune Thrombocytopenia in Chronic Lymphocytic Leukemia. J Blood Disorders Transf 5: 198. doi: 10.4172/2155-9864.1000198

Page 3 of 4

\begin{tabular}{|c|c|c|c|c|c|c|c|c|c|}
\hline 43. & 71 & $\mathrm{~F}$ & C & 10.4 & 43.0 & 103 & 0.96 & 2.6 & 2.5 \\
\hline 44. & 59 & $M$ & B & 11.6 & 54.0 & 352 & 0.87 & 2.8 & 7.8 \\
\hline 45. & 73 & M & C & 9.6 & 44.1 & 96 & 0.52 & 3.2 & 2.4 \\
\hline 46. & 57 & $M$ & B & 11.8 & 48.3 & 294 & 0.78 & 3.5 & 5.6 \\
\hline 47. & 77 & $M$ & C & 10.8 & 60.2 & 84 & 0.81 & 3.4 & 2.2 \\
\hline 48. & 53 & $\mathrm{~F}$ & A & 11.5 & 29.4 & 384 & 1.01 & 2.6 & 7 \\
\hline 49. & 69 & $M$ & C & 10.0 & 46.1 & 112 & 0.75 & 2.8 & 3.1 \\
\hline 50. & 61 & $\mathrm{~F}$ & A & 11.6 & 27.8 & 343 & 0.98 & 2.5 & 11.2 \\
\hline 51. & 53 & $M$ & B & 12,1 & 78.3 & 274 & 0.94 & 3.6 & 6.8 \\
\hline 52. & 77 & $\mathrm{~F}$ & A & 11.4 & 45.6 & 232 & 0.65 & 3.2 & 5.4 \\
\hline 53. & 64 & $\mathrm{~F}$ & A & 11.7 & 33.4 & 345 & 0.78 & 2.4 & 13.1 \\
\hline 54 & 66 & $\mathrm{~F}$ & C & 9.5 & 50.2 & 78 & 0.84 & 2.9 & 3.2 \\
\hline 55. & 50 & $M$ & B & 11.6 & 43.6 & 236 & 0.89 & 3.0 & 6.9 \\
\hline 56. & 80 & $M$ & C & 10.3 & 48.9 & 94 & 0.74 & 3.4 & 2.1 \\
\hline 57. & 55 & $M$ & B & 11.9 & 37.8 & 324 & 0.98 & 3.8 & 4.6 \\
\hline 58. & 75 & $M$ & C & 10.0 & 41.2 & 88 & 0.66 & 2.8 & 2.8 \\
\hline 59. & 61 & $\mathrm{~F}$ & B & 11.2 & 43.6 & 245 & 0.48 & 3.5 & 5.9 \\
\hline 60. & 64 & $M$ & A & 11.9 & 56.0 & 324 & 0.78 & 2.6 & 10.2 \\
\hline 61. & 69 & $\mathrm{~F}$ & C & 9.8 & 40.1 & 72 & 0.82 & 3.2 & 3.5 \\
\hline 62. & 66 & $M$ & B & 11.2 & 47.6 & 263 & 0.56 & 3.6 & 4.5 \\
\hline 63. & 73 & $M$ & C & 10.2 & 30.1 & 91 & 0.72 & 3.0 & 2.3 \\
\hline 64. & 57 & $\mathrm{M}$ & B & 12.6 & 47.3 & 326 & 0.75 & 3.5 & 5.3 \\
\hline 65. & 76 & $M$ & C & 9,7 & 53.2 & 79 & 0.68 & 3.3 & 2.6 \\
\hline 66. & 54 & $M$ & A & 12.5 & 25.7 & 394 & 0.66 & 2.4 & 17 \\
\hline 67. & 70 & $M$ & C & 10.5 & 37.0 & 93 & 0.56 & 2.9 & 3.3 \\
\hline 68. & 61 & $\mathrm{~F}$ & A & 11.4 & 28.9 & 325 & 1.02 & 2.8 & 12.2 \\
\hline 69. & 69 & $\mathrm{~F}$ & B & 12.1 & 34.9 & 348 & 0.88 & 3.4 & 6.1 \\
\hline 70. & 60 & $M$ & B & 12.2 & 49.3 & 347 & 0.96 & 3.3 & 6.3 \\
\hline 71. & 72 & $M$ & C & 10.1 & 45.1 & 91 & 0.78 & 2.6 & 2.8 \\
\hline 72. & 58 & $\mathrm{~F}$ & A & 11.9 & 34.2 & 324 & 1.02 & 2.4 & 17 \\
\hline 73. & 56 & $M$ & B & 12.4 & 48.9 & 353 & 0.78 & 2.9 & 5.1 \\
\hline 74. & 74 & $\mathrm{~F}$ & C & 9.4 & 48.4 & 89 & 0.56 & 3.0 & 2.1 \\
\hline 75. & 75 & $M$ & C & 10.9 & 44.8 & 104 & 0.52 & 2.9 & 2.6 \\
\hline 76. & 55 & $M$ & B & 12.4 & 46.6 & 348 & 0.65 & 3.4 & 6.1 \\
\hline 77. & 70 & $M$ & C & 10.6 & 40.2 & 106 & 0.54 & 3.1 & 3.1 \\
\hline 78. & 62 & $\mathrm{~F}$ & A & 12.2 & 28.4 & 325 & 0.76 & 3.2 & 5.4 \\
\hline 79 & 60 & $\mathrm{~F}$ & A & 11.9 & 34.6 & 389 & 0,98 & 2.6 & 11.4 \\
\hline 80 & 68 & $\mathrm{~F}$ & C & 9.9 & 45.3 & 82 & 0.82 & 3.3 & 3.5 \\
\hline 81. & 78 & $M$ & C & 10.0 & 45.4 & 85 & 0.57 & 3.2 & 2.7 \\
\hline 82. & 52 & $M$ & B & 12.4 & 34.9 & 354 & 0.78 & 3.6 & 5.3 \\
\hline 83. & 57 & $\mathrm{~F}$ & A & 11.8 & 26.9 & 389 & 0.99 & 2.6 & 10.8 \\
\hline 84 & 73 & $M$ & C & 9.1 & 45.8 & 78 & 0.48 & 2.8 & 2.9 \\
\hline
\end{tabular}

Table 1: Characteristics of patients with CLL.

cases $\mathrm{CVP}=19$ cases, Fludarabine alone $=10$ cases, $\mathrm{RFC}=8$ cases, alemtuzumab $=4$ cases. Immune thrombocytopenia was present in six patients (7.1\%). In all cases, it appeared during the evolution of CLL. One patient $(1.2 \%)$ associated autoimmune hemolytic anemia (hemoglobin value $=8 \mathrm{~g} / \mathrm{dl}$, reticulocytes $=3 \%$, test Coombs positive, $\mathrm{LDH}=384 \mathrm{u} / \mathrm{L}$, indirect bilirubin $=1.8 \mathrm{mg} / \mathrm{dl}) .23 \%$ of patients with CLL had unmutated IgVH (1.2\% associated with ITP, $21.8 \%$ without ITP). The patients with CLL-stage $\mathrm{C}$ and immune thrombocytopenia were treated with corticosteroids alone in four cases and corticosteroids and chemotherapy in two cases, switching from stage $\mathrm{C}$ in stage $\mathrm{A}$ in three cases and stage $B$ in one case. The patients with $C$ stage infiltrative (25 cases) were treated with chemotherapy and the switch from stage $\mathrm{C}$ to stage B was realised in three cases. All patients had at diagnosis low FORD values (between 0.39-0.96 mmol/L) and high FORT values (between 2.5-3.8 mmol/L) (Table 2). There are no significant differences in the level of oxidative stress between CLL patients with or without ITP. The bone marrow infiltration with lymphocytes was higher at patients with CLL-stage C infiltrative (84\%) versus patients with CLL-stage C immune (49\%). The median follow up for patients in both groups was of three years. The median overall survival of patients with CLL-stage A was 11.2 years, CLL-stage B was 5.7 years and CLL-stage C was 3.2 years. In patients with CLL-stage $C$ immune overall survival rate was of 5.7 years versus 2.8 years in patients with CLL-stage C infiltrative.

The latest studies of autoimmune cytopenia in CLL revealed an incidence ranging between 4.5 and $10 \%[3,4,10]$. In our small study, the prevalence was of $7 \%$ and in agreement with these studies.

Old age, male sex, a high absolute lymphocyte count, advanced stage of disesase, purine analogs treatment have been associated with autoimmune cytopenia and a high bone marrow infiltration and elevated $\beta 2$ microglobuline with CLL-stage C infiltrative compared to CLL -stage C immune $[2,4,11]$. Our data showed an old age, male sex and a high blood absolute lymphocyte count higher in patients with CLL and immune thrombocytopenia versus patients without immune 
Citation: Gaman AM,Gaman MA (2014) Immune Thrombocytopenia in Chronic Lymphocytic Leukemia. J Blood Disorders Transf 5: 198. doi: 10.4172/2155-9864.1000198

\begin{tabular}{|c|c|c|c|}
\hline & $\begin{array}{c}\text { CLL-stage } \mathrm{C} \text { immune } \\
\text { (6 patients) }\end{array}$ & \begin{tabular}{|c|c|} 
CLL-stage C infiltrative \\
(25 patients)
\end{tabular} & p \\
\hline Median age ( years) & 68 & 73 & NS \\
\hline Male (\%) & 66 & 70 & NS \\
\hline $\mathrm{Hb} \times 10^{9} / \mathrm{L}$ & 10.4 & 10 & NS \\
\hline WBC $\times 10^{9} / \mathrm{L}$ & 34.2 & 45.1 & NS \\
\hline $\begin{array}{l}\text { absolute Ly count } \\
\times 10^{9} / \mathrm{L}\end{array}$ & 18.6 & 25.4 & NS \\
\hline Platelet count $\times 10^{9} / \mathrm{L}$ & 86 & 91 & NS \\
\hline $\begin{array}{l}\text { Bone marrow infiltration } \\
\%\end{array}$ & 49 & 84 & 0.05 \\
\hline $\begin{array}{l}\beta 2 \text { microglobuline } \\
>2.5 \mathrm{mg} / \mathrm{L} \%\end{array}$ & 50 & 70 & 0.01 \\
\hline FORD values (mmol/L) & $0.39-0.84$ & $0.41-0.96$ & NS \\
\hline FORT values (mmol/L) & $2.7-3.8$ & $2.5-3.4$ & NS \\
\hline Overall survival, years & 5.7 & 2.8 & 0.01 \\
\hline
\end{tabular}

Table 2: Characteristics of patients with CLL-stage $C$ immune disease versus CLLstage $\mathrm{C}$ infiltrative disease.

thrombocytopenia, but not statistically significant. An increased bone marrow infiltration and a high $\beta 2$ microglobuline level were significantly higher in patients with CLL-stage C infiltrative compared to CLL-stage C immune.

The mechanisms underlying immune thrombocytopenia in chronic lymphocytic leukemia are still unclear; nevertheless, the immune system is perturbed by the patient's underlying condition before the onset of immune thrombocytopenia [12] and is significantly associated with unmutated IgVH and the development of autoimmune hemolytic anemia [5]. Oxidative stress is involved in the perturbation of the immune response and might be involved in the development of CLL. On the other hand, in the secondary immune thrombocytopenia from HIV and HCV infections, it was revealed that specific antibodies for GP49-66 have the ability to induce reactive oxygen species leading to complement-independent platelet fragmentation [5,9]. We tried to evaluate the level of oxidative stress in patients with CLL and establish if CLL is an oxidative stress related disease or whether only CLL patients with ITP have any defects of the imbalance of oxygen metabolism. All patients had low FORD values (between $0.39-0.96 \mathrm{mmol} / \mathrm{L}$ ) and high FORT values (between $2.5-3.8 \mathrm{mmol} / \mathrm{L}$ ) and there were no significant differences of oxidative stress between patients with CLL and immune thrombocytopenia compared to patients with CLL without immune thrombocytopenia. Microarray data specifying gene difference in oxygen stress between CLL with and without ITP were not available.

The effect of immune thrombocytopenia on the clinical outcome and survival of patients with CLL is controversial $[2,4,5]$. Some studies revealed a poor survival rate at patients with CLL and immune thrombocytopenia than other patients with CLL, and this effect appears independently of the common clinical prognostic variables $[4,5]$. Other studies showed that the development of autoimmune cytopenia at any time during the evolution of the disease did not influence prognosis significantly [10]. The patients with CLL-stage C immune disease had a significantly better survival rate than the patients with CLL-stage C infiltrative disease (median survival rate of 7.4 years versus 3.7 years)

[10], data which was also confirmed by our results (median survival rate of 5.7 years in patients with CLL-stage $\mathrm{C}$ immune disease versus 2.8 years in patients with CLL-stage $\mathrm{C}$ infiltrative disease). These results may be the effect of the response to corticosteroid therapy in immune thrombocytopenia and of the shifting to an early stage of disease.

In our short group of patients, oxidative stress did not play a role in the appearance of immune thrombocytopenia in CLL. Our small number of patients has not enough power to get conclusive results for the contribution of oxidative stress in the development of ITP in CLL patient; therefore, future research on a bigger group of patients is necessary for a significant conclusion. The patients with CLL-stage C immune disease had a longer survival rate than patients with CLL-stage $\mathrm{C}$ infiltrative disease.

\section{References}

1. Chiorazzi N, Rai KR, Ferrarini M (2005) Chronic lymphocytic leukemia. N Eng J Med 352: 804-815

2. Mauro FR, Foa R, Cerretti R, Giannarelli D, Coluzzi S, et al. (2000) Autoimmune hemolytic anemia in chronic lymphocytic leukemia: clinical, therapeutic, and prognostic features. Blood 95: 2786-2792.

3. Dearden C (2008) Disease-specific complications of chronic lymphocytic leukemia. Hematology Am Soc Hematol Educ Program.

4. Visco C, Ruggeri M, Laura Evangelista M, Stasi R, Zanotti R, et al. (2008) Impact of immune thrombocytopenia on the clinical course of chronic lymphocytic leukemia. Blood 111: 1110-1116.

5. Stasi R (2011) Immune pathophysiology of primary immune therombocytopenia Hematology Eur Hematol Assoc Educ Program 173-178.

6. Halliwell B, Gutteridge JMC (1998) Free radicals in biology and medicine Oxford University Press, London.

7. Knight JA (1999) Free Radicals, Antioxidants, Aging and Disease, AACC Press Washington DC, USA.

8. Valko M, Leibfritz D, Moncol J, Cronin MT, Mazur M, et al. (2007) Free radicals and antioxidants in normal physiological functions and human disease. Int $J$ Biochem Cell Biol 39: 44-84.

9. Nardi M, Feinmark SJ, Hu L, Li Z, Karpatkin S (2004) Complement-independent $\mathrm{Ab}$-induced peroxide lysis of platelets requires 12-lipoxygenase and a platelet NADPH oxidase pathway. J Clin Invest 113: 973-980.

10. Moreno C, Hodgson K, Ferrer G, Elena M, Filella X, et al. (2010) Autoimmune cytopenia in chronic lymphocytic leukemia: prevalence, clinical associations, and prognostic significance. Blood 116: 4771-4776.

11. Barcellini W, Capalbo S, Agostinelli RM, Mauro FR, Ambrosetti A, et al. (2006) Relationship between autoimmune phenomena and disease stage and therapy in B-cell chronic lymphocytic leukemia. Haematologica 91: 1689-1692.

12. Johnsen $\mathrm{J}$ (2012) Pathogenesis in immune thrombocytopenia: new insights. Hematology Am Soc Hematol Educ Program 2012: 306-312. 